\title{
Comparison of Heritabilities Estimated by Two Techniques of Variance Analysis for Fry Mortality Rate of Masu Salmon in Hokkaido
}

\author{
Yasuji Kanno*1 and Hiroshi Kawamura*2 \\ (Received November, 30, 1992)
}

\begin{abstract}
Two methods of heritability estimation for threshold character were compared to clarify merits and demerits. The data on mortality in the fry stage of masu salmon originating from six mature females and ten mature males caught in the Shokanbetsu River in Hokkaido were used. The heritabilities were estimated by two methods, i.e. the coding method where 0 or 1 values are assigned to dead and alive fry, and the arcsine transformation method where percentage data of dead fry obtained from lots of two series of experiments for six half sibs as to distinct females and ten half sibs as to distinct males are used. From the results two points can be emphasized. One is that heritabilities for initial mortality rate are minute and almost zero, suggesting the action of strong environmental influences. The other is that the coding method can be used to estimate the heritability instead of the arcsine transformation method for threshold character.
\end{abstract}

To discover the heritability as the efficiency of inheritance of traits from parents to offspring is important in order to estimate the selection effectiveness of fish populations in aquaculture. The parameter offers most fundamental information for the genetic improvement of races of fish as well as information about biotechnological techniques in which recently significant advancements have been made.

In such quantitative characteristics as body length and body weight, the methods of heritability estimation are already established. In this case, the analysis of variance are used as an important technique of heritability estimation. Meanwhile, the heritability of traits such as the threshold character of dead and alive individuals is usually estimated by the method of arcsine transformation of the percentage of a set of data. However, since this method requires more than two experimental crowds from fry in the same crossing combination, the difficulty in extension of experimental design and in maintaining the same environmental control over all rearing sections is virtually increased. On account of this shortcoming, little literature concerning the estimation of heritability of threshold character has been published as yet.

The coding method where 0 or 1 values are as- signed to each of two attributes in the threshold character was used for the first time by Kanis et al. ${ }^{1}$ ) for mortality in egg and larval stages of three species of genus Salmo. Though there are many merits in the coding method compared to the arcsine transformation method, the techniques of calculation have not been explained in detail until now. So researchers of fish breeding do not understand or utilize it.

In the present paper the authors compare the heritabilities of ry mortality estimated by using two techniques, i.e. the coding method and the arcsine transformation method, and propose using the coding method to a heritability estimation of allor-none traits such as dead and alive fry.

\section{Materials and Method}

The materials were 2,890 fry derived from crossing between six females and ten males of masu salmon Oncorhynchus masou caught in the Shokanbetsu River, Hokkaido. Specifically, the materials comprise two series of fry from which ten groups of eggs from a female were fertilized by the sperm of ten males and six groups of eggs from six fernales were fertilized by the sperm of one male. The fry which hatched in each egg group in January, 1991 were divided into two

*1 Faculty of Fisheries, Hokkaido University, Hakodate 041, Japan (栺野泰次：北海道大学水産学部)，

*2 Hokkaido Fish Hatchery Makkari Branch, Makkari, Hokkaido 048-16, Japan (河村 博: 北海道立 水産尒化場真狩支場). 
lost of equal number and reared under uniform conditions in running water in a wooden tank $(35 \times 350 \times 30 \mathrm{~cm})$. Water temperature ranged from 6 to $8^{\circ} \mathrm{C}$. The fry were regularly fed pellets twice a day. Dead fry until 31st March 1991 were counted and the mortality rates were calculated for every lot.

There are two important assumptions in estimating heritabilities using quantitative data. One is that the structure of measurements is described as the additive model: $x_{i j}=\mu+\alpha_{i}+\varepsilon_{i j}$, where $x_{i y}$ is the measurement of character, $\mu$ the population mean, $\alpha_{i}$ the effect of $i$ th parent and $\varepsilon_{i j}$ the error effect by chance, and another is that the distribution of error terms is conformed to the normal distribution $N\left(0, \sigma^{2}\right)$ on all levels of $\alpha_{i}$. The arcsine transformation method $\left(\sin ^{-1} \sqrt{y}\right)$ of percentage data $(y)$ is a sort of approximation which was proposed to satisfy the above two assuptions when the threshold character is given as two attaributes in a partitional table. ${ }^{2)}$ Similarly, the coding method where 0 or 1 values are assigned for two attributes of threshold phenomena is also an approximation, and so the estimates of heritability by the coding method contain some bias owing to the unsatisfied second assumption.

The arcsine transformation method is not noted here, since ordinary calculating techniques were applied. But the coding method is described in detail because few examples of estimation of heritability have been applied to this method, and the techniques of calculation have not been written in detail until now.

\section{Results}

Analysis of Variance of Data Series Composed of 0 and I Values

Nowadays many threshold characteristics are considered to be polygenetic. Of those characteristic, the death of larval fish may be caused by the additive effect of polygenes which weaken the resistance of fish to a bad environment. ${ }^{2}$. The analysis of variance to threshold characteristics called the coding method here is carried out by assigning 0 or 1 values to two phenotypic attributes. The development of the formula has been described as follows by Taguchi. ${ }^{\text {? }}$

When $p$ is an occurrence probability of 0 , and $q(=1-p)$ is that of 1 value, the variance of the data series of 0 and 1 is given as $p(1-p)$ per unit datum, and the total sum of squares is $N p(1-p)$. Now, let the data or 0 or 1 be variable $x_{i j}$, the occurrence frequency of 1 be $r$, and the total number of data be $N$. So, the resolution of the total sum of squares of data is as follows.

$$
\begin{aligned}
\mathrm{SS}_{T}=N p(1-p)= & N \frac{r}{N}\left(1-\frac{r}{N}\right) \\
= & r-\frac{r^{2}}{N} \\
= & \left(x_{1}{ }^{2}+x_{2}{ }^{2}+\cdots+x_{n}{ }^{2}\right) \\
& -\frac{\left(x_{1}+x_{2}+\cdots+x_{n}\right)^{2}}{N}
\end{aligned}
$$

The form of formula (1) for resolving the total sum of squares coincides with the formula for resolving general quantitative data. Therefore, we can analyze the data series by the same manner as in the quantitative variable.

Data arranged in a one way layout used in the

\begin{tabular}{|c|c|c|c|c|c|c|}
\hline \multirow{2}{*}{ Individual } & \multicolumn{5}{|c|}{ Female parent } & \multirow{2}{*}{ Total } \\
\hline & $q_{1}$ & $q_{2}$ &. & $\cdot$ & $q_{L}$ & \\
\hline 1 & $x_{11}$ & $x_{12}$ & . & . & $x_{1 L}$ & \\
\hline 2 & $x_{21}$ & $x_{22}$ & $\cdot$ & . & $x_{2 L}$ & \\
\hline$\cdot$ & $\cdot$ & $\cdot$ & $\cdot$ & $\cdot$ & $\cdot$ & \\
\hline$\cdot$ & $\cdot$ & $\cdot$ & · & $x_{i j}$ & · & \\
\hline . & $\cdot$ & $\cdot$ & & & $\cdot$ & \\
\hline$n$ & $x_{m 1}$ & $x_{n 2}$ & $\cdot$ & $\cdot$ & $x_{n L}$ & \\
\hline Total & $\sum_{i} x_{i 1}$ & $\sum_{i} x_{i 2}$ & . & - & $\sum_{i} x_{i L}$ & \\
\hline Number of data & $n_{1}$ & $n_{2}$ & . & $\cdot$ & $n_{L}$ & $N$ \\
\hline
\end{tabular}
present study is shown in Table 1. The number

Table 1. Measurement structure in a case in which eggs from $L$ females were fertilized by the sperm of one male. Data comprise the values of 0 or 1 
Table 2. Numbers of dead and alive fry in the respective two lots of each crossing section from hatching to March 31 1991, and their mortality rate

\begin{tabular}{|c|c|c|c|c|c|c|c|c|}
\hline \multicolumn{2}{|c|}{ Parents } & \multicolumn{2}{|c|}{ Lot 1} & \multirow{2}{*}{$\begin{array}{l}\text { Mortality } \\
\text { rate }\end{array}$} & \multicolumn{2}{|c|}{ Lot 2} & \multirow{2}{*}{$\begin{array}{l}\text { Mortality } \\
\text { rate }\end{array}$} & \multirow{2}{*}{$Z_{0}$} \\
\hline Female & Male & Dead & Alive & & Dead & Alive & & \\
\hline 1 & 1 & 7 & 93 & 0.070 & 11 & 88 & 0.111 & $1.011 *$ \\
\hline 2 & 1 & 22 & 78 & 0.220 & 6 & 94 & 0.060 & $3.261^{*}$ \\
\hline 3 & 1 & 24 & 76 & 0.240 & 11 & 89 & 0.110 & $2.419 *$ \\
\hline 4 & 1 & 15 & 85 & 0.150 & 7 & 93 & 0.070 & 1.808 \\
\hline 5 & 1 & 16 & 84 & 0.160 & 7 & 93 & 0.070 & $1.995^{*}$ \\
\hline 6 & 1 & 21 & 79 & 0.210 & 3 & 97 & 0.030 & $3.917 *$ \\
\hline 1 & 1 & 7 & 93 & 0.070 & 11 & 88 & 0.111 & 1.011 \\
\hline 1 & 2 & 10 & 79 & 0.112 & 7 & 81 & 0.080 & 0.741 \\
\hline 1 & 3 & 1 & 99 & 0.010 & 8 & 92 & 0.080 & $2.388^{*}$ \\
\hline 1 & 4 & 3 & 79 & 0.037 & 17 & 65 & 0.207 & $3.341^{*}$ \\
\hline 1 & 5 & 0 & 88 & 0.000 & 13 & 74 & 0.149 & $3.769 *$ \\
\hline 1 & 6 & 6 & 71 & 0.078 & 8 & 68 & 0.105 & 0.586 \\
\hline 1 & 7 & 10 & 71 & 0.123 & 5 & 75 & 0.063 & 1.330 \\
\hline 1 & 8 & 0 & 79 & 0.000 & 7 & 71 & 0.300 & $2.724^{*}$ \\
\hline 1 & 9 & 15 & 85 & 0.150 & 23 & 77 & 0.230 & 1.442 \\
\hline 1 & 10 & 1 & 52 & 0.019 & 4 & 48 & 0.077 & 1.397 \\
\hline
\end{tabular}

$Z_{0}$ are statistics to test for equality of mortality rates between two lots.

* Having significant difference at $5 \%$ level.

of fry from every female parent may be unequal. If we pay attention to $x_{i j}=x_{i, j}^{2}$, the total sum of squares

$$
\mathrm{SS}_{T}=\sum_{j=1}^{L} \sum_{i=1}^{n_{j}} x_{i j}-\frac{\left(\sum_{j=1}^{L} \sum_{i=1}^{n_{j}} x_{i j}\right)^{2}}{N}
$$

the sum of squares between parents combination

$$
\mathrm{SS}_{\mathrm{B \theta \textrm {t }}}=\sum_{j=1}^{L} \frac{\left(\sum_{i=1}^{n_{j}} x_{i j}\right)^{2}-\left(\sum_{j=1}^{L} \sum_{i=1}^{n_{j}} x_{i j}\right)^{2}}{N}
$$

and the sum of squares between individuals

$$
\mathbf{S S}_{e}=\mathbf{S S}_{r}-\mathbf{S S}_{\mathrm{Bet}}
$$

where $L$ is the number of female parents, and $n_{j}$ is the number of fry from the $j$ th fermale parent. In coding 0 or 1 for two categorical attributes, even if either attribute is 1, the results of the calculation remain unchanged.

Estimation of Heritability in Larval Mortality by Means of Coding Method

Data for dead fry originating from the Shokanbetsu River (Table 3) were analyzed by means of analysis of variance using the coding method, and the estimates of heritability $\left(V_{G} / V_{P}\right)$ in a narrow sense are shown in Table 4 (Female) and Table 5 (Male). The process of calculation is
Table 3. Numbers of dead and alive fry in each

\begin{tabular}{|c|c|c|c|c|c|}
\hline \multicolumn{2}{|c|}{ Parents } & \multicolumn{2}{|c|}{ Number of fry } & \multirow{2}{*}{ Total } & \multirow{2}{*}{$\begin{array}{l}\text { Mortality } \\
\text { rate }\end{array}$} \\
\hline Femael & Male & Dead & Alive & & \\
\hline 1 & 1 & 18 & 181 & 199 & 0.090 \\
\hline 2 & 1 & 28 & 172 & 200 & 0.140 \\
\hline 3 & 1 & 35 & 165 & 200 & 0.175 \\
\hline 4 & 1 & 22 & 178 & 200 & 0.110 \\
\hline 5 & 1 & 23 & 177 & 200 & 0.115 \\
\hline 6 & 1 & 24 & 176 & 200 & 0.120 \\
\hline 1 & 1 & 18 & 181 & 199 & 0.090 \\
\hline 1 & 2 & 17 & 160 & 177 & 0.096 \\
\hline 1 & 3 & 9 & 191 & 200 & 0.045 \\
\hline 1 & 4 & 20 & 144 & 164 & 0.122 \\
\hline 1 & 5 & 13 & 162 & 175 & 0.074 \\
\hline 1 & 6 & 14 & 139 & 153 & 0.092 \\
\hline 1 & 7 & 15 & 146 & 161 & 0.093 \\
\hline 1 & 8 & 7 & 150 & 157 & 0.045 \\
\hline 1 & 9 & 38 & 162 & 200 & 0.190 \\
\hline 1 & 10 & 5 & 100 & 105 & 0.050 \\
\hline
\end{tabular}
crossing section from hatching to March 31 1991, and their mortality rates

shown in detail below the variance tables. Estimates of heritability are minute and almost zero; $h_{F}{ }^{2}=0.011$ for females, and $h_{M}{ }^{2}=0.076$ for males.

Estimation of Heritability by Means of the Arcsine Transformation Method

In comparison with the mortality rates between 
Table 4. Variance table and heritability for females calculated by the coding method

\begin{tabular}{crrrrr}
\hline Factor & SS & df & $\mathrm{ms}$ & $F_{0}$ & $E(\mathrm{~ms})$ \\
\hline $\begin{array}{c}\text { Between females } \\
\text { error }\end{array}$ & 0.8525 & 5 & 0.1705 & 1.56 & $\sigma_{c}{ }^{2}+k \sigma_{f}{ }^{2}$ \\
\hline
\end{tabular}

(1) Calculation of sum of squares

$$
\left\{\begin{array}{l}
\mathrm{CF}=\frac{\left(\sum_{j=1}^{L} \sum_{i=1}^{n} x_{i j}\right)^{2}}{N}=\frac{(1049)^{2}}{1199}=917.766 \\
\mathrm{SS}_{T}=\sum_{j=1}^{L} \sum_{i=1}^{n} x_{i j}-\mathrm{CF}=1049-917.766=131.234 \\
\mathrm{SS}_{f}=\sum_{j=1}^{L} \frac{\left(\sum_{i=1}^{n} x_{i j}\right)^{2}}{n_{j}}-\mathrm{CF}=\frac{(181)^{2}}{199}+(172)^{2}+\cdots+\frac{(176)^{2}}{200}-917.766 \\
=0.852
\end{array}\right.
$$

$\mathrm{SS}_{e}=\mathrm{SS}_{\bar{I}}-\mathrm{SS}_{f}=131.234-0.852=130.382$

(2) Calculation of $k$

$$
k=\frac{1}{(L-1)}\left(N-\frac{\sum_{j=1}^{L} n_{j^{2}}}{N}\right)=\frac{1}{5}(1199-199.834)=199.83
$$

(3) Calculation of heritability $\left(h_{F}{ }^{2}\right)$

$$
\left\{\begin{array}{l}
\sigma_{f}^{2}=\frac{m s_{f}-m s_{e}}{k}=\frac{0.1705-0.1093}{199.83}=0.000306 \\
\sigma_{e}^{2}=0.1093 \\
h_{F}^{2}=\frac{4 \sigma_{f}^{2}}{\sigma_{f}^{2}+\sigma_{e}^{2}}=\frac{4 \times 0.000306}{0.000306+0.1093}=0.0112
\end{array}\right.
$$

Table 5. Variance table and heritability for males calculated by the coding method

\begin{tabular}{cccccc}
\hline Factor & $\mathrm{SS}$ & $\mathrm{df}$ & $\mathrm{ms}$ & $F_{0}$ & $E(\mathrm{~ms})$ \\
\hline $\begin{array}{c}\text { Between males } \\
\text { error }\end{array}$ & 3.1279 & 9 & 0.3475 & $4.22^{*}$ & $\sigma_{\mathrm{c}}{ }^{2}+k \sigma_{m}{ }^{2}$ \\
$\sigma_{e}{ }^{2}$
\end{tabular}

* Significant at $1 \%$ level.

(1) Calculation of sum of squares

$\mathrm{CF}={\mathrm{(1535)^{2 }}}_{1691}=1393.391$

$\mathrm{SS}_{T}=1535-1393.391=141.609$

$\mathrm{SS}_{m}={ }_{189}+{ }_{171)^{2}}^{(160)^{2}}+\cdots+{ }_{100)^{2}}-1393.391=3.128$

$\mathrm{SS}_{e}=141.609-3.128=138.481$

(2) Calculation of $k$

$k=\frac{1}{9}(1691-173.539)=168.607$

(3) Calculation of heritability $\left(h_{\mu^{2}}\right)^{2}$

$\sigma_{\mathrm{m}}^{2}=\frac{0.3475-0.0824}{168.61}=0.00157$

168.61

$\sigma_{*}{ }^{2}=0.0824$

\begin{tabular}{|c|c|c|c|c|c|}
\hline Factor & SS & df & $\mathrm{ms}$ & $F_{0}$ & $E(\mathrm{~ms})$ \\
\hline $\begin{array}{c}\text { Between females } \\
\text { error }\end{array}$ & $\begin{array}{l}0.008768 \\
0.046501 \\
\end{array}$ & $\begin{array}{l}5 \\
6 \\
\end{array}$ & $\begin{array}{l}0.00175 \\
0.00775 \\
\end{array}$ & 0.23 & $\begin{array}{l}\sigma_{e}^{2}+2 \sigma_{f}^{2} \\
\sigma_{e}^{2}\end{array}$ \\
\hline \multicolumn{6}{|c|}{$\begin{array}{l}\sigma f^{2}=\frac{0.00175-0.00775}{2}=-0.006 \\
\sigma_{\varepsilon}^{2}=0.00775 \\
h_{F}^{2}=\frac{4 \times(-0.006)}{-0.006+0.00775+0.25}=-0.0953\end{array}$} \\
\hline Factor & SS & $\mathrm{df}$ & $\mathrm{ms}$ & $F_{0}$ & $E(\mathrm{~ms})$ \\
\hline $\begin{array}{c}\text { Between males } \\
\text { error }\end{array}$ & $\begin{array}{l}0.03487 \\
0.04123 \\
\end{array}$ & $\begin{array}{r}9 \\
10 \\
\end{array}$ & $\begin{array}{l}0.00387 \\
0.00412 \\
\end{array}$ & 0.94 & $\begin{array}{l}\sigma_{e}^{2}+2 \sigma_{m}^{2} \\
\sigma_{e}^{2}\end{array}$ \\
\hline $\begin{array}{l}\sigma_{\pi^{2}}^{2}=\frac{0.00387-0.004}{2} \\
\sigma_{\ell}^{2}=0.00412 \\
h_{M}^{2}=\frac{4 \times(-0.0}{-0.00025+0.0}\end{array}$ & $\frac{0025}{25}=-0.003$ & & & & \\
\hline
\end{tabular}

$4 \times 0.0016$

$h_{M}^{2}=\frac{4 \times 0.0016}{0.0016+0.0824}=0.0762$

Table 6. Variance table and heritability for females (above) and males (below) calculated by the arcsine transformation method 
two lots, significant differences at the $5 \%$ level were recognized in four combinations for female and male series, respectively (Table 2 ). The results of estimation of heritability by means of analysis of variance of the arcsine transformation data for each mortality rate of fry from two lots (Table 2) are shown in Table 6. The constant value of 0.25 in the formula to estimate heritability is the variance component among individuals where mortality rates were transformed by radian. The estimates of heritability are nearly zero with negative signs for male $h_{F}{ }^{2}=-0.095$ and for male $h_{M}{ }^{2}=-0.004$. Those negative signs are theoretically meaningless. Therefore, the heritability in the population is guessed to be zero.

\section{Discussion}

Generally speaking, it is thought to be pertinent that the heritability of threshold character is calculated by means of the arcsine transformation method. To apply this method. however, the mortality rates of two or more lots from the brood of a parental combination must be used. The reason is that the mortality rate of a lot is analyzed as one datum after transformation and data from the experiment composed of one lot group cannot calculate variance in error terms. In the rearing experiment using plural numbers of lost, great difficulties arise in keeping the uniformity of experimental conditions, a large scale of facilities is necessary to get an accurate estimate, and such experiments are expensive. On the other hand, the coding method can be applied to experiments which consist of one lot group, but the method does not seem to be so precise as the arcsin transformation method because the assumption required in error terms of the model to estimate heritability, $x_{i j}=\mu+\alpha_{i}+\varepsilon_{i j}$, is not fully satisfied.

The coding method has two defects; one is that the distribution of error terms is not normal, and the other is that variance $p(1-p)$ per datum in each rearing lot is not uniform throughout all the lots. In spite of having those theoretical defects, however, the coding method has some advantages when the method is compared with the arcsine transformation method. Firstly it is not necessary to divide a brood into two or more lots. Secondly, it is possible to calculate genetic correlations with other quantitative characteristics. The threshold characteristic controlled by polygenes may often correlate with some other quan- titative characteristics due to the linkage of relevant genes. Such relationships are roughly estimated as genetic correlations with other quantitative characters where the coding method is used, whereas it cannot be calculated by the arcsine transformation method.

Kanis et $a .^{13}$ reported that heritability for the mortality rate of the larval stage in three species of Salmonidae is $0.01-0.15$ for Atlantic salmon Salmo salar, 0.01-0.06 for sea trout Salmo trutta, and 0.06-0.14 for rainbow trout Salmo gairdneri. In the present study, the heritability of larval mortality rates for masu salmon is 0.011 for females and 0.076 for males by the coding method. Those are in a range of that estimated by Kanis et al. This suggests that a low heritability in the mortality of the larval stage is universal in a species such as Salmonidae.

The heritability for females and males calculated by the arcsine transformation method had values with negative signs, despite theoretically never taking place. Kanis et al. reported negative signs of heritability and ascribed the cause to a mixture of errors in the experimental treatments. In the present study, a comparison of mortality rates between two lots from a brood showed that the number of pairs with significant differences was more numerous than the number explained as the effect of the type I statistical error. Although to carry out rearing experiments under uniform conditions is ideal, the actual rearing conditions of lots were not always to be maintained in the same state. Those non-uniform conditions must have brought about the difference in mortality rates between lots, and resulted in obtaining the values of heritability with negative signs. It is not realistic that the arcsine transformation method is superior to the coding method.

Two points are emphasized in the present paper. One is that the heritability of larval mortality rate is minute and almost zero, suggesting the action of strong environmental influences. Therefore, there is no efficiency for improving mortality rate at the larval stage. The other is that the values of heritability calculated by means of the two methods resembled each other. This suggests that the coding method can be used to estimate the heritability for threshold character instead of the arcsine transformation method. The coding method is useful in cases where maintenance of homogeneous experimental conditions is difficult. 


\section{Acknowledgements}

The authors express their sincere thanks to Dr. Tetsuichiro Kinoshita, associate professor of Hokkaido University, for his critical reading of the manuscript. The present work was supported in part by a Grant-in-Aid for Scientific Research (C) from The Ministry of Education, Science, and Culture of Japan.

\section{References}

1) E. Kanis, T. Refstie, and T, Gjedrem: A genetic analysis of egg, alvin and fry mortality in salmon (Salmo salar), sea trout (Salmo trutta) and rainbow trout (Salmo gairdneri). Aquaculture, 8, 259-268 (1976).

2) T. P. Bogyo and W. A. Becker: Estimates of heritability from transformed percentage sib data with unequal subclass numbers. Biometrics, 21, 1001-1008 (1965).

3) D. S. Falconer: Introduction to quantitative genetics. 3rd ed., John Wiley \& Sons, New York, 1989, pp. 300-312.

4) G. Taguchi: Tokei Kaiseki, 2nd ed., Maruzen, Tokyo, 1972, pp. 107-135. (in Japanese) 